\title{
Percepción y consumo de plantas medicinales en los profesionales de enfermería, hospitales red asistencial-EsSalud, Amazonas - 2018.
}

\section{Perception and consumption of medicinal plants in nursing professionals, hospitales red asistencial-EsSalud, Amazonas - 2018.}

\author{
Yshoner Antonio Silva Díaz; ; Luz Edith Aguilar Guevara²
}

\section{RESUMEN}

El presente trabajo de investigación de enfoque cuantitativo fue de nivel descriptivo, de corte transversal prospectivo; se realizó con el objetivo de diagnosticar la percepción y consumo de plantas medicinales en los profesionales de enfermería, Hospitales Red Asistencial-EsSalud, Amazonas-2018. La muestra estuvo constituida por 61 profesionales de enfermería. Se utilizó como instrumentos: el cuestionario de percepción y de consumo de las plantas medicinales. Los resultados: existe relación altamente significativa sobre la percepción y consumo de las plantas medicinales por los profesionales de enfermería de la Red Asistencia EsSalud Amazonas (X2=4,33; $\mathrm{Gl}=4 ; \mathrm{p}=0.649>0.05$ ); la percepción es buena con el $69.4 \%$ y un alto consumo con un $85.5 \%$; el consumo de plantas medicinales por los profesionales de enfermería según el sexo masculino es alto con el $32 \%$ y en las mujeres es de 52\%. Las conclusiones: la percepción de las plantas medicinales de los profesionales de enfermería existe una percepción buena y regular. Los profesionales de enfermería de los hospitales de la Red Asistencial Amazonas - EsSalud, presentan un consumo alto de las plantas medicinales; tanto los varones y las mujeres muestran buena percepción sobre las plantas medicinales, de igual forma existe un alto consumo

Palabras clave: percepción, consumo, plantas medicinales, profesionales de enfermería y hospitales.

\begin{abstract}
The present work of investigation of quantitative approach was of descriptive level, of prospective transversal cut; was carried out with the objective of diagnosing the perception and consumption of medicinal plants in nursing professionals, Red Asistencial Hospitals-EsSalud, Amazonas-2018. The sample consisted of 61 nursing professionals. We used as instruments: the questionnaire of perception and consumption of medicinal plants. The results: there is a highly significant relationship on the perception and consumption of medicinal plants by the nursing professionals of the EsSalud Amazonas Assistance Network $(\mathrm{X} 2=4.33$, G1 = 4, p = 0.649>0.05); perception is good with $69.4 \%$ and high consumption with $85.5 \%$; The consumption of medicinal plants by nursing professionals according to the male sex is high with $32 \%$ and in women it is $52 \%$. The conclusions: the perception of medicinal plants of nursing professionals there is a good and regular perception. The nursing professionals of the hospitals of the Amazonas Assistance Network - EsSalud, have a high consumption of medicinal plants; Both men and women show good perception about medicinal plants, in the same way there is a high consumption.
\end{abstract}

Keywords: perception, consumption, medicinal plants, nursing professionals and hospitals.

\footnotetext{
'Magister, Docente de la Facultad de Ciencias de la Salud; Director del Departamento Académico de Salud Pública de la Facultad de Ciencias de la Salud de la Universidad Nacional Toribio Rodríguez de Mendoza de Amazonas.Email: yshoner@hotmail.com

${ }^{2}$ Licenciada en Enfermería. Enfermera Asistencial del Hospital I Higos Urco-EsSalud Chachapoyas. Email: luzedith82@hotmail.com
} 


\section{INTRODUCCIÓN}

La percepción y el consumo de las plantas medicinales en las personas, ha venido siendo utilizado como medicina alternativa para aliviar los problemas de salud, por lo que más del $75 \%$ de las personas utilizan las plantas medicinales. En Europa, se estimó el mercado anual en el uso de las plantas medicinales en 7000 millones de euros. En España muy a la cola de otros países europeos como Alemania o Francia, según el informe de resultados de la "Investigación sobre los hábitos de consumo de plantas con fines terapéuticos en España" publicado por Infito (Centro de Investigación sobre Fitoterapia) en, 1 de cada 3 personas $(32,8 \%)$ consume plantas con fines terapéuticos. (Alonso, 2015).

Desde la eclosión de los fármacos de síntesis que forma la base de la terapéutica oficial de los países occidentales, las plantas de uso medicinal han seguido teniendo, no obstante, un lugar principal en el desarrollo de la farmacología. Se calcula que existen en el mundo más de 250 mil especies vegetales; de entre ellas se consideran como potencialmente medicinales unas 12 mil especies, pero debe tenerse en cuenta que solo se tiene conocimiento científico de un 10\% del total de las especies (Alonso, 2015).

El consumo de plantas medicinales con fines terapéuticos en la población española por comunidades autónomas. Entre ellos cabe destacar el perfil del consumidor y el nivel de conocimientos, que se define mayoritariamente como el de una mujer (74,6\%), de clase media (media: $42,8 \%$ media alta: $22,8 \%$ media baja: $34,4 \%$ ) y de edad madura (36 a 50 años: $32,9 \%$, > de 50 años: $45,4 \%$ ). En cuanto a la tradición de uso, 1 de cada 4 consumidores $(25 \%)$ reconoce tradición de uso familiar o en su entorno, y entre los consumidores, 1 de cada 3 (29\%) tiene consumo diario, dándose la mayor frecuencia de consumo diario entre las mujeres y a medida que avanza la edad.

El consumo de las plantas medicinales en el Perú es tan antiguo como nuestra cultura andina, muchos conocimientos se encuentran arraigados en el saber popular, sin embargo, la excesiva "modernización" de la Medicina Occidental ha hecho que estos conocimientos sean relegados, y en ciertos aspectos, hasta olvidados. Dentro de este contexto, el uso de las plantas medicinales ha sido y seguirá siendo una alternativa preventiva y curativa para la salud, pero como todo recurso natural está sujeto a la extracción indiscriminada porsimple recolección y su consumo no estará garantizado mientras no existan normas que aseguren su sanidad y calidad. (Comisión de Ambiente, Ecología. Congreso de la República del Perú, 2013).

En la Región Amazonas, desde su descubrimiento ha existido siempre una diversidad cultural, tal es así por ejemplo, el 34\% de la población total de amazonas utilizan diferentes tipos de medicina tradicional y rituales para el tratamiento de las enfermedades. Existen comunidades nativas en las provincias de Bagua y Condorcanqui habitan en los distritos selváticos de Bagua, Aramango e Imaza. Poseen su propio idioma y dialecto y una singular artesanía y utilizan las plantas medicinales de diversas formas y con diferentes utilidades de acuerdo a la variedad de plantas que existen en cada parte de la Región Amazonas. Son representantes de este grupo humano los aguarunas y los huambisas y representan $23 \%$ de la población total del Departamento de Amazonas y el $6 \%$ corresponde a personas adultas mayores. (INDES, 2014)

El perfil del personal de la salud debería ser: haber nacido y crecido en el boom de las nanotecnologías, adaptados al cambio tecnológico, pero tendrán que enfrentar dilemas éticos complejos, cambios en las terapéuticas, exigencias de especialización, dominio de idiomas, competencias interculturales y uso de la investigación" (Castrillón, 2014).

Considerando esta realidad problemática a nivel internacional, nacional y local se planteó el siguiente problema de investigación: ¿Cuál es la percepción y consumo de plantas medicinales en los profesionales de enfermería, Hospitales Red Asistencial-EsSalud, Amazonas - 2018?, teniendo en cuenta que cada civilización tiene sus propias plantas curativas o medicinales.

La percepción y el efecto curativo de la medicina tradicional normalmente se basan en la aplicación de diferentes hierbas medicinales y otros productos de la naturaleza (tierra, agua, productos de origen animal, etc.) con frecuencia en un rito especial que solamente conoce el curandero o médico tradicional. Durante la evolución de la humanidad se desarrolló un conocimiento profundo del efecto curativo de las plantas dentro de cada civilización. Conocemos por ejemplo la medicina tradicional China, la medicina Ayurveda de la India y el curanderismo de Latinoamérica. La eficacia de la medicina tradicional normalmente no es transferible, sino está ligada a la civilización donde se desarrolló. Si tratamos las plantas medicinales como recursos genéticos, podemos afirmar que existe una riqueza botánica enorme, especialmente en los países andinos. Hasta 
hoy día, el campesino o la «hierbera» recolecta las plantas medicinales del campo. Pero conforme pasa el tiempo muchas especies ya son escasas, principalmente aquellas de las cuales se utilizan las raíces. Las especies vegetales curativas por naturaleza son recursos naturales renovables, esto significa que tienen la capacidad de reproducirse tanto sexualmente como vegetativamente y de esta manera mantener el stock natural de manera constante a través del tiempo.

Asimismo, no existe garantía sobre la procedencia, porque muchas plantas medicinales pueden haber sido recogidas de zonas cercanas basurales, desagües, carreteras o acequias infestadas por diversos parásitos. Muchas plantas medicinales cultivadas como la "manzanilla", el "toronjil" y la "menta" entre otras especies, provienen de campos contaminados por agroquímicos (fertilizantes sintéticos, pesticidas, herbicidas y fungicidas).

Por lo tanto se planteó como objetivo general determinar la percepción y consumo de plantas medicinales en los profesionales de enfermería, Hospitales Red Asistencial-EsSalud, Amazonas 201 8, con la finalidad de realizar un diagnóstico de la percepción y consumo de las plantas medicinales por parte de los profesionales de enfermería que laboran en los hospitales de la Red Asistencial Amazonas.

En los siguientes capítulos de la tesis se presentan los objetivos, el marco teórico utilizado para la investigación, los materiales y métodos, los resultados y discusión así como las conclusiones y recomendaciones.

\section{MATERIAL Y MÉTODOS}

Estuvo conformado por todos los profesionales de enfermería de los Hospitales de EsSalud de la Red Asistencial Amazonas: Hospital I Higos Urco (25), Hospital El Buen Samaritano Utcubamba (18), Hospital Héroes del Cenepa (18), hicieron un total de 61.

\section{Criterios de inclusión:}

- Licenciados de enfermería de ambos sexos que desearon participar de manera voluntaria de la presente investigación.

- Licenciados de enfermería que laboraron en los Hospitales de la Red Asistencial EsSalud Amazonas. - Licenciados de enfermería que laboraron en el área asistencial y administrativa.

- Licenciados de enfermería que laboraron en los diferentes servicios asistenciales.

\section{Criterios de Exclusión:}

- Licenciados de enfermería de ambos sexos que no desearon participar de la presente investigación.

- Licenciados de enfermería que no laboraron en EsSalud de la Red Asistencial Amazonas.

- Licenciados de enfermería que se encontraron con Licencia sin goce.

\section{Métodos:}

Método: El método que se utilizó fue la encuesta (Hernández, 1994).

Técnica e instrumentos de recolección de datos. Para medir la variable en estudio se utilizó la técnica del cuestionario, que ayudó a la recopilación de datos a través de ítems tipo Likert (Hernández, 2010).

\section{Instrumento:}

Para obtener la información básica a fin de cumplir con los objetivos propuestos, para la variable 1: percepción de los profesionales de enfermería de las plantas medicinales se utilizó la guía de cuestionario, que consta de 25 ítems; las respuestas fueron según la escala ordinal de tipo Likert, donde $1=$ nunca; $2=$ casi nunca; $3=$ A veces; $4=$ casi siempre y $5=$ siempre.

Para la variable 2: consumo de las plantas medicinales se utilizó como instrumento la guía de cuestionario con una escala de medición de tipo ordinal (Likert) elaborado por el autor; se identificó el consumo de las plantas medicinales en los profesionales de enfermería de los hospitales de la Red Asistencial Amazonas-EsSalud-2018, que constó de 2 dimensiones, se aplicó la guía de cuestionario tipo likert, donde los licenciados en enfermería tuvieron que marcar según su criterio nunca, casi nunca, a veces, casi siempre y siempre. El tiempo para el llenado del instrumento fue de 10 minutos. Se asignó un puntaje tal como se detalla.

\section{RESULTADOS}

Figura 1: Percepción y consumo de plantas medicinales en los profesionales de enfermería, Hospitales Red Asistencial-EsSalud, Amazonas 2018.

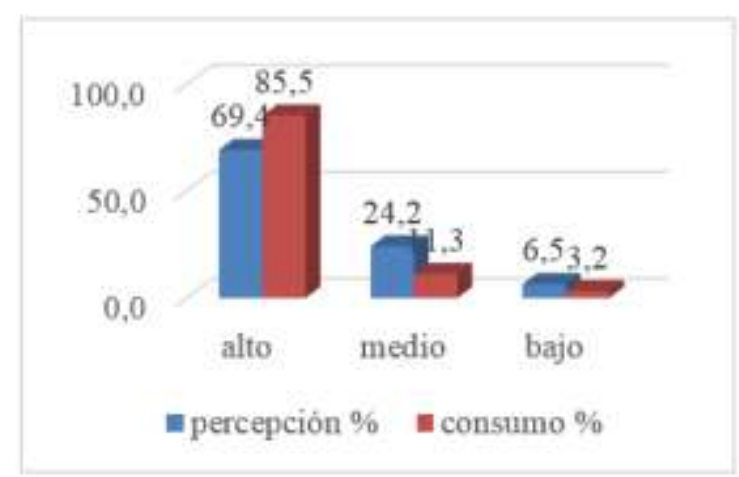


Figura 2: Diagnostico de la percepción de plantas medicinales en los profesionales de enfermería, Hospitales Red Asistencial-EsSalud, Amazonas2018.

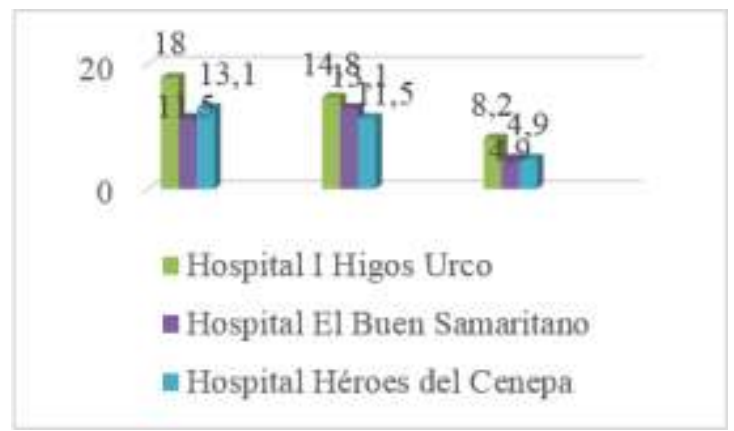

Figura 3: Diagnostico del consumo de plantas medicinales en los profesionales de enfermería, Hospitales Red Asistencial-EsSalud, Amazonas2018.

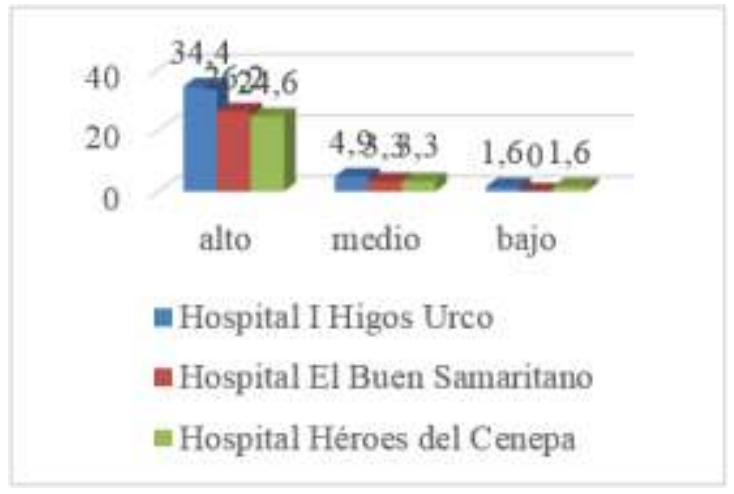

Figura 4: Diagnóstico de consumo de plantas medicinales en los profesionales de enfermería, según sexo, Hospitales Red Asistencial-EsSalud, Amazonas-2018.

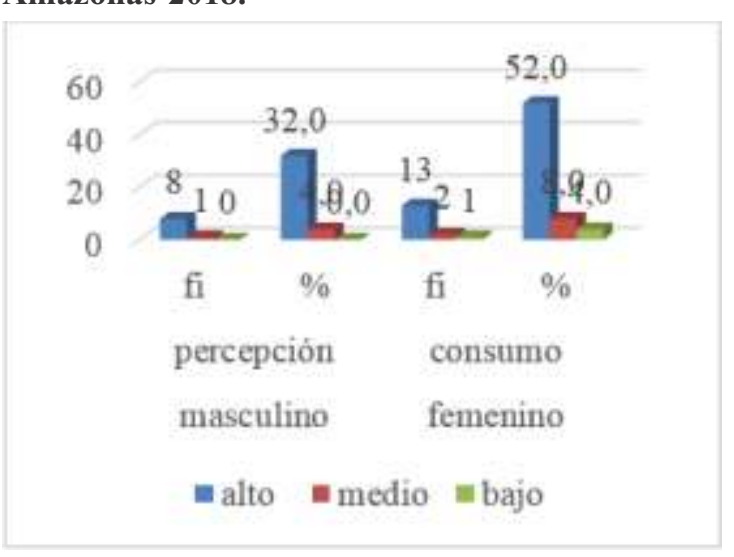

\section{DISCUSIÓN}

En la tabla y/o figura 01, se evidencia que del 100\% de las personas que se les aplicó la guía de cuestionario, el $69.4 \%$ presentaron buena percepción de las plantas medicinales y el $85 \%$ presentaron un alto consumo de las mismas. Se afirma la hipótesis alterna: $\mathrm{X} 2=4,33 ; \mathrm{Gl}=4 ; \mathrm{p}=0.649$ $>0.05$.

Al respecto, Fernández (2014), en Chile; en su trabajo de investigación cuyo objetivo fue: determinar la percepción tienen las personas sobre el uso de las plantas medicinales en la comuna de Nueva Imperial. Los resultados: un $80 \%$ usa las plantas medicinales como infusión (a beber).

Se puede afirmar que tanto el consumo como la percepción sobre las plantas medicinales, es positivo tanto para los profesionales de enfermería y para todas las personas; guarda relación los resultados de Fernández con los nuestros donde se refleja que éstas se consumen de diferente manera, realizándose su consumo de diversas maneras para aliviar el dolor, tratar infecciones, como desinflamantes, etc.

En la tabla y/o figura 02, se obtiene por resultado, respecto al diagnóstico de la percepción de las plantas medicinales de los profesionales de enfermería que existe una percepción buena y regular.

Clement, (2015); en Trinidad Venezuela. En su investigación cuyo objetivo fue: evaluar la percepción de eficacia de las plantas medicinales por los usuarios acceder a los servicios de salud primaria de todo el territorio de Trinidad. Resultados: 265 usuarios a base de plantas entraron en el estudio y cita más de 100 hierbas para la promoción de la salud / bienestar y la gestión de determinados problemas de salud. El ajo es el más popular de hierbas (en el 48,3\% de la muestra) y se utilizó para el resfriado común, tos, fiebre, como "limpieza de sangre" y carminatives. También se utilizó en un $20 \%$ de pacientes con hipertensión. 230 usuarios $(86,8 \%)$ indicaron que las hierbas son eficaces y la percepción de que habían igual o mayor eficacia que los medicamentos alopáticos convencionales. Género, etnicidad, ingresos y años de educación formal no haya influido en los pacientes la percepción de la eficacia de hierba, pero sí la edad $(p=0,036)$. El uso concomitante de hierbas y medicamentos alopáticos es relativamente alto en el $30 \%$, y la mayoría de los usuarios no informó a su médico tratante. Conclusión: la mayoría de los usuarios tienen la percepción de que las hierbas son eficaces, y en algunos casos, más eficaz que los medicamentos convencionales. Sugerimos que esta percepción puede ser un importante factor que influye en la sostenida y creciente popularidad de las hierbas.

Al respecto se evidencia que existe coincidencia con los resultados obtenidos, esto debido a que se afirma 
que existe buena percepción respecto al uso de las plantas medicinales, y es que necesariamente debemos aceptar que en nuestro medio se utiliza las plantas medicinales en su mayoría para el tratamiento de diversas enfermedades, claro que se debería manejar el tema de la dosificación y la planta adecuada específicamente para tratar cierta enfermedad. En EsSalud se ha implementado a nivel nacional el área de medicina alternativa donde existen profesionales médicos y no médicos entrenados para recetar medicina complementaria en el tratamiento de distintos males de las personas aseguradas, esto definitivamente se realiza porque existe buena percepción de las plantas medicinales y sus resultados positivos en el tratamiento de las mismas.

En la tabla y/o gráfico 03 , se obtiene como resultado: que en el Hospital I Higos Urco Chachapoyas el $34,4 \%$ presentan un consumo alto de las plantas medicinales, continuado por el Hospital el Buen Samaritano de Utcubamba donde los profesionales de enfermería presentan un consumo alto $(26,2 \%)$ y los profesionales de enfermería del Hospital Héroes del Cenepa-Bagua presentan de igual forma un consumo elevado (24,6\%).

En consecuencia, Aguilar (2017), en Chile, en su investigación: consumo que tienen las personas sobre el uso de las plantas medicinales en la comuna de Nueva Imperial. Los resultados del proyecto un $80 \%$ usan las plantas medicinales como infusión (a beber). De esto un gran número las prepara de una manera inadecuada lo que implica muchas veces quitarles sus propiedades curativas e incluso se corre el riesgo de intoxicación. Un amplio porcentaje de los encuestados manifestó interés por la masificación de este conocimiento, sobre todo en la correcta preparación y elaboración de productos naturales basados en plantas medicinales recolectados en nuestra comuna. Otro grupo importante $(22 \%)$ opina que faltan lugares donde aprender técnicas relacionadas con medicina herbaria. En relación al conocimiento de las Plantas medicinales; se puede concluir que las personas conocen hasta 15 plantas para remedio, pero desconocen una preparación adecuada para mejorar enfermedades más comunes.

Chávez y Roldán, (1994), refiere que en cuanto a la tradición de consumo, 1 de cada 4 consumidores $(25 \%)$ reconoce tradición de uso familiar o en su entorno. Asimismo las encuestas realizadas en 2006 para la elaboración del Libro Blanco de los herbolarios dieron como principal razón de los usuarios para el consumo de "medicina natural" el consejo familiar, con un $56 \%$ de respuestas.
Al respecto, se puede manifestar que existe en todos - los ámbitos similitud respecto al consumo de las plantas medicinales, si bien es cierto, en algunas ocasiones los profesionales de enfermería niegan el uso de las mismas con los mismos pacientes, pero realmente es que cada profesional de alguna manera siempre utilizan y/o consumen las plantas medicinales.

El consumo de plantas medicinales en el Perú es tan antiguo como nuestra cultura andina, muchos conocimientos se encuentran arraigados en el saber popular, sin embargo, la excesiva "modernización" de la Medicina Occidental ha hecho que estos conocimientos sean relegados y en ciertos aspectos, hasta olvidados. Dentro de este contexto, el uso de las plantas medicinales ha sido y seguirá siendo una alternativa preventiva y curativa para la salud, pero como todo recurso natural está sujeto a la extracción indiscriminada porsimple recolección y su consumo no estará garantizado mientras no existan normas que aseguren su sanidad y calidad. (Comisión de Ambiente, Ecología. Congreso de la República del Perú, 1999).

En éste sentido coincide con lo que refiere la Comisión de Ambiente, Ecología del Congreso de la República; el uso de las plantas medicinales son usadas para diferentes tipos de enfermedades infecciosas, respiratorias urinarias, gastrointestinales, parasitarias ya sea de manera externa como interna, aunque en muchas veces no se maneje bien el tema de la dosificación, la conservación y todo lo que tenga que ver sobre la calidad del consumo.

\section{CONCLUSIONES}

- Existe relación altamente significativa entre percepción y consumo de los profesionales de enfermería de los hospitales de la Red Asistencial Amazonas, EsSalud - 2018.

- Respecto al diagnóstico de la percepción de las plantas medicinales de los profesionales de enfermería existe una percepción buena y regular.

- Los profesionales de enfermería de los hospitales de la Red Asistencial Amazonas - EsSalud, presentan un consumo alto de las plantas medicinales.

- Los profesionales del sexo masculino y femenino muestran buena percepción sobre las plantas medicinales, de igual forma existe un alto consumo.

\section{REFERENCIAS BIBLIOGRÁFICAS}

Annan Y Houghton Bonilla E. 2013. El 
Conocimiento de la Realidad Social. Más allá del dilema de los métodos. Editorial Presencia. Bogotá, Colombia. 29 p.

Alonso, Galarza, Glave, Grande. 2013. Amazonas: Lineamientos Estratégicos de DesarrolloPlan Binacional de Desarrollo de la Región Fronteriza Perú - Ecuador, Capítulo, Perú. Págs. 141-144.

Barthelson Berlin, B. 2017. Bases Empíricas de la Cosmología Botánica Aguaruna. En: Etnicidad y Ecología. CAAAP. Lima, Perú.

Boucher F., Riveros H. 2013. Agroindustria y agroindustria rural: elementos conceptuales y de reflexión, Serie Documentos de Trabajo PRODAR No. 12. Lima, Perú, 45p.

Comisión Ambiental Regional de Amazonas, Instituto de Investigaciones de la Amazonía Peruana. 2017. Estrategia Regional de la Diversidad Biológica de Amazonas - Biodamaz. Convenio de Cooperación Técnica Internacional entre Perú y Finlandia: Proyecto Diversidad Biológica de la Amazonía Peruana BIODAMAZ. Pág. 87.

Comisión de Ambiente, Ecología. Congreso de la República del Perú. 2017. Dictamen Ley de Aprovechamiento Sostenible de Plantas Medicinales. Lima, 12 de mayo de 2017. Pág. 132.

Congreso de la República del Perú. 2013. Ley 27.300. Pe/Aprovechamiento sostenible de las plantas medicinales. Promulgada el 07.Julio.2000. Publicada el 08. Julio.2000.

Chez Ra, Jonas Wb. 2017. The challenge of complementary and alternative medicine. Am J Obst Gynecol.

Instituto de Investigaciones Alexander Von Humboldt, 2013. Los Viveros de plantas nativas. Cultivando nuestras semillas, conservando la biodiversidad. Taller Manejo de viveros de especies nativas, restauración ecológica y planificación del paisaje rural.

Muller Jl, Clauson Ka. 2017. Pharmaceutical considerations of common herbal medicine. Am J Managed Care.

Naccarato, P. 2017. Los Aguarunas del Alto Marañón. Una breve Aproximación a las Condiciones de Vida de Comunidades Ribereñas de la Provincia de Condorcanqui, Amazonas. Documento de trabajo. Lima, Perú.

Saipe, Aeci, Alboan y Cipca, 2017. Evaluación Participativa de Necesidades Prioritarias en el Alto Marañón, Región Amazonas.
Gutiérrez Alvarado W. 2017. Ponencia: Recursos Naturales en la Amazonia. II Jornada Binacional de Químicos Farmacéuticos y Bioquímicos Perú-Ecuador. VIII Expo Farmacia Internacional 2006. Iquitos, Perú.

Romero Bonilla H. 2013. Memoria de Segundo Congreso Internacional de Plantas Medicinales. Universidad Técnica de Machala, RELIPLAM. Ecuador.

Yon de Prentice M. 2013. Ponencia: Los Recursos Naturales en el Desarrollo de la Región. II Jornada Binacional de Químicos Farmacéuticos y Bioquímicos PerúEcuador. VIII Expo Farmacia Internacional 2006. Iquitos, Perú.

Campos Jiménez S., Esquivel Blanco., Chacón Montero M. 2012. Proyecto de plantas medicinales colegio técnico profesional de Cóbano. Colegio Técnico Profesional de Cóbano, Instituto Nacional de biodiversidad de Costa Rica.

Ramírez Vargas Rosa. 2012. Catálogo de las Plantas Diuréticas de la Zona Norte del Perú. Memorias del II Congreso Internacional de Plantas Medicinales. 2012. RELIPLAM, Universidad Técnica de Machala. Ecuador.

Fundación para la Innovación Agraria. 2012. Diversificación en la producción de plantas medicinales. Boletín de Plantas Medicinales y Aromáticas. Santiago de Chile. Boletín Trimestral N5, Septiembre 2002.

Barrón Araoz R. 2017. La comunidad andina, mercado estratégico para los productos agroindustriales peruanos. Quipukamayoc: Segundo Semestre 1999.Lima.

Revista de Enfermería. El cuidado cultural: una alternativa frente al cuidado de la salud de la población en condición de DFV. 